\title{
HYBRID COMPUTATION OF NORMAL MODE TUNE SHIFTS IN ROUNDED-RECTANGULAR PIPES*
}

\author{
V. Galdi, D.I.I.I.E. - Univ. of Salerno, I-84084 Fisciano (SA), Italy \\ $\underline{\text { S. Petracca }}^{\dagger \ddagger}$, and I. M. Pinto, Univ. of Sannio at Benevento, I-82100 Benevento, Italy
}

\begin{abstract}
A fast and accurate hybrid (analytical-numerical) technique for computing the normal mode tune-shifts in rounded-rectangular (stadium) pipes is introduced based on Galerkin method together with a smart representation of Poisson's equation Green's function in a rectangular domain. Comparison with standard finite-elements and finite difference methods shows that our method is faster and more accurate, requiring no numerical differentiation.
\end{abstract}

\section{THE PROBLEM}

Many beam-pipe cross-section geometries of potential interest for accelerators, including the stadium-shaped one recently proposed for LHC [3], differ from the rectangle only by the rounding of corners, or the substitution of straight sides with circular arcs. Computing the related betatron tune-shifts, resulting from collective (space-charge and image) effects is a key problem to prevent resonant betatron excitations leading to potentially harmful beam instabilities. The normal mode coherent and incoherent ${ }^{1}$ tune-shifts can be written in terms of the normal mode Laslett coefficients $\epsilon$ as follows [2]:

$$
\Delta \nu=-\frac{N R r_{0}}{\pi \nu \beta_{0}^{2} \gamma_{0} L^{2}} \epsilon
$$

where ${ }^{2}$ :

$$
\begin{aligned}
\epsilon_{1,2} & =\frac{L^{2}}{4 \Lambda}\left\{-\frac{\delta_{x} \partial_{x} \Phi^{(i m)}+\delta_{y} \partial_{y} \Phi^{(i m)}}{2}+\right. \\
\pm & {\left[\left(\frac{\delta_{y} \partial_{y} \Phi^{(i m)}-\delta_{x} \partial_{x} \Phi^{(i m)}}{2}\right)^{2}+\right.} \\
& \left.\left.+\delta_{x} \partial_{x} \Phi^{(i m)} \delta_{y} \partial_{y} \Phi^{(i m)}\right]^{1 / 2}\right\}
\end{aligned}
$$

$\Phi^{(i m)}$ is the image-potential produced in the beam pipe by a linear charge density $\Lambda$ going through the beam center of charge $\underline{r}_{b}, N$ is the number of particles in the beam, $R$ is

\footnotetext{
* Work supported in part by INFN.

$\dagger$ On leave of absence from KEK, Tsukuba, JPN.

$\ddagger$ e-mail stefania@kekvax.ac.jp

${ }^{1}$ The incoherent and coherent regimes correspond to $\underline{r} \neq \underline{r}_{b}=\underline{r}_{e q}$ and $\underline{r}=\underline{r}_{b} \neq \underline{r}_{e q}$, respectively, $\underline{r}_{e q}$ denoting the beam center of charge equilibrium position [2].

${ }^{2}$ The pipe-shape independent space-charge contribution to the tuneshift is neglected here for simplicity.
}

the machine radius, $r_{0}$ is the classical particle radius, $L$ is a scaling length (usually, the maximum pipe diameter), $\nu$ is the nominal tune, and

$$
\left\{\begin{array}{l}
\delta_{x, y}=\left.\partial_{x, y}\right|_{\underline{r}=\underline{r}_{b}}, \text { incoherent regime, } \\
\delta_{x, y}=\left.\left(\partial_{x, y}+\partial_{x_{b}, y_{b}}\right)\right|_{\underline{r}=\underline{r}_{b}}, \text { coherent regime. }
\end{array}\right.
$$

\section{THE METHOD}

For computing the image potential $\Phi^{(i m)}$ in rounded rectangular geometries, it is convenient to use the rectangulardomain Green's function $g_{R}$ (henceforth RDGF), viz. :

$$
\begin{gathered}
\Phi^{(i m)}\left(\underline{r}, \underline{r}_{b}\right)=\Phi\left(\underline{r}, \underline{r}_{b}\right)-\Lambda g_{0}\left(\underline{r}, \underline{r}_{b}\right), \\
\Phi\left(\underline{r}, \underline{r}_{b}\right)=\Lambda\left[\sum_{k} \int_{\sigma_{k}} g_{R}\left(\underline{r}, l_{k}\right) \rho_{\sigma_{k}}\left(l_{k}\right) d l_{k}+g_{R}\left(\underline{r}, \underline{r}_{b}\right)\right],
\end{gathered}
$$

where $g_{0}$ is the free-space Green's function, the unknown $\rho_{\sigma_{l}}$ are obviously nonzero only on the rounded portion of $\partial S_{0}$, i.e., the $\operatorname{arcs} \sigma_{k}$, and $l_{k}$ is the arc-length on $\sigma_{k}$.

We seek a hybrid (analytical-numerical) solution of eq. (4) by using Galerkin (moments) method [6], whereby we first expand the unknown $\rho_{s}$ :

$$
\rho_{\sigma_{k}}\left(l_{k}\right)=\sum_{n=1}^{N} b_{n}^{(k)} w_{n}^{(k)}\left(l_{k}\right),
$$

into a suitable (finite) set of basis functions $\left\{w_{1}^{(k)}\left(l_{k}\right), \ldots, w_{N}^{(k)}\left(l_{k}\right)\right\}$, defined on $\sigma_{k}$, where $\left\{b_{1}^{(k)}, \ldots, b_{N}^{(k)}\right\}$, are $N$-dimensional vectors of unknown coefficients, and then enforce the (Dirichlet) boundary conditions on the $\operatorname{arcs} \sigma_{k}$, whence:

$$
\begin{gathered}
\int_{\sigma_{k}} \Phi\left(l_{k}, \underline{r}_{b}\right) w_{n}^{(k)}\left(l_{k}\right) d l_{k}=0, \\
n=1,2, \ldots, N ; k=1,2, \ldots, P,
\end{gathered}
$$

thus obtaining a block-matrix linear system:

$$
[\mathbf{L}] \mathbf{b}=\mathbf{c} .
$$

The matrix $[\mathbf{L}]$ is readily shown to be symmetrical, positive definite and hence non-singular. The components of $\mathbf{b}, \mathbf{c}$ and $\mathbf{L}$ are explicitly given by (5),

$$
c_{i}^{(k)}=-\int_{\sigma_{k}} w_{i}^{(k)}\left(l_{k}\right) g\left(l_{k}, \underline{r}_{b}\right) d l_{k},
$$




$$
i=1,2, \ldots, N ; k=1,2, \ldots, P,
$$

and:

$$
\begin{gathered}
{\left[L_{M}^{(p, q)}\right]_{i j}=\int_{\sigma_{p}} \int_{\sigma_{q}} g\left(l_{p}, l_{q}\right) w_{i}^{(p)}\left(l_{p}\right) w_{j}^{(q)}\left(l_{q}\right) d l_{p} d l_{q}} \\
i, j=1,2, \ldots, N, \quad p, q=1,2, \ldots, P
\end{gathered}
$$

where the upper indexes identify the block sub-matrix, and the lower ones the element in each sub-matrix.

Using eq.s (2)-(5), once (7) has been solved, the Laslett coefficients can be computed without resorting to numerical differentiation. This makes the proposed method definitely more accurate than both finite-differences and finiteelements.

\section{IMPLEMENTATION AND COMPUTATIONAL BUDGET}

Fast and accurate numerical solution of (7) follows from a skillful choice of the RDGF representation in (4) and the basis functions in (5).

A rapidly converging series expansion of the RDGF [5] , which explicitly contains the (logarithmic) singular term is ${ }^{3}$ :

$$
g_{R}\left(\underline{r}, \underline{r}_{b}\right)=-\sum_{m=-\infty}^{\infty} \log \frac{T_{m}^{10}\left(\underline{r}, \underline{r}_{b}\right) T_{m}^{01}\left(\underline{r}, \underline{r}_{b}\right)}{T_{m}^{00}\left(\underline{r}, \underline{r}_{b}\right) T_{m}^{11}\left(\underline{r}, \underline{r}_{b}\right)},
$$

where:

$$
\begin{gathered}
T_{m}^{p q}\left(\underline{r}, \underline{r}_{b}\right)=1+\exp \left[-2\left|y-(-)^{p} y_{b}+2 b m\right| \frac{\pi}{a}\right]+ \\
-2 \exp \left[\left|y-(-)^{p} y_{b}+2 b m\right| \frac{\pi}{a}\right] \cos \left[\frac{\pi}{a}\left(x-(-)^{q} x_{b}\right)\right],
\end{gathered}
$$

$a, b$ being the rectangle side lengths.

A convenient set of (partially overlapping) piece-wise parabolic subdomain basis functions, can be defined in terms of the local angles $\phi$ (we drop the suffix $k$ for simplicity) as follows:

$$
\begin{gathered}
w_{i}(\phi)=\frac{\Delta \phi^{2}-\left(\phi-\phi_{i}\right)^{2}}{\Delta \phi^{2}}, \\
\phi_{i}-\Delta \phi\left(1-\delta_{i 1}\right) \leq \phi<\phi_{i}+\Delta \phi\left(1-\delta_{i N}\right), \\
i=1,2, \ldots, N,
\end{gathered}
$$

where $\Delta \phi$ is the angular discretization step (assumed the same for all arcs), $\phi$ is related to the local arc-length $l$ by $l=R \phi, R$ being the local curvature radius, and $\delta_{r s}$ is the Kronecker symbol ${ }^{4}$. The relevant local coordinate systems are sketched in Fig. 1. Note that: $i$ ) the choice of subdomain basis functions, rather than full-domain ones, results

\footnotetext{
${ }^{3}$ It is easily recognized that the (logarithmic) singularity of $g_{R}$ appears in the $T_{0}^{10}$ term.

${ }^{4}$ For $i=1, N$, eq. (12) yields the correct behaviour at the points where the circular arcs join the straight portions of $\partial S_{0}$, where $\rho_{s}$ can be different from zero, but its derivative should vanish.
}

into fewer singular integrals in $[\mathbf{L}]$; ii) no polygonal approximation of the arcs is implied, resulting into fewer functions being needed for a given accuracy.

Letting $P$ the number of arcs in the rounded portion of $\partial S_{0}$, the system (7) has rank $N P$. Computing the matrix elements requires evaluating up to $P N(P N-1) / 2$ double-integrals ${ }^{5}$. These latter can be either evaluated numerically using standard routines appropriate for regular [7] and singular integrands [8], or analytically [4]. Matrix inversion for solving (7) is not the most demanding task, in view of the typically small $(N P \approx 20) \mathbf{L}$ matrix size. In all numerical simulations below we truncated (10) at $|m| \leq 3$ and took $\Delta \phi=\pi / 10$, corresponding to a matrix size $N P=20$.

\section{NUMERICAL RESULTS AND CONCLUSIONS}

The circular pipe, for which the tune-shifts are known exactly, is the hardest conceivable test case for the proposed method (largest departure from rectangular geometry). It is seen from Fig. 2 that the obtained accuracy is very good.

Our method was subsequently applied [4] to a number of different proposed geometries relevant to LHC [3].

As an example the contour-level plots for the incoherent and coherent (both normal modes) Laslett coefficients for a stadium-shaped pipe, sketched in Fig. 3, are shown in Fig.s 4-6.

As a conclusion, we found that the above hybrid approach is comparatively faster and more accurate than available finite-element and/or finite-difference techniques.

\section{REFERENCES}

[1] L.J. Laslett, Proc. of the 1963 Summer Study BNL 7534.

[2] S. Petracca, Particle Accel., 48, 81, 1994.

[3] The LHC study group, CERN/AC/95-05 (LHC), 1995.

[4] V. Galdi, S. Petracca, I. Pinto, Particle Accel., PA(R)-85, 1999, in print.

[5] M. Bressan and G. Conciauro, Alta Frequenza, LII, 188, 1983, ibidem LVII, 217, 1988.

[6] R.F. Harrington, Field Computation by Moment Methods, McMillan, New York, 1961.

[7] W.H. Press et al., Numerical Recipes, the Art of Scientific Computing, 2nd Ed., Cambridge Un. Press, 1992.

[8] SLATEC Pub. Dom. Math. Lib., NETLIB, http://www.netlib.org

\footnotetext{
${ }^{5}$ Due to geometrical (specular) symmetries, the effective number of elements to compute is usually smaller.
} 


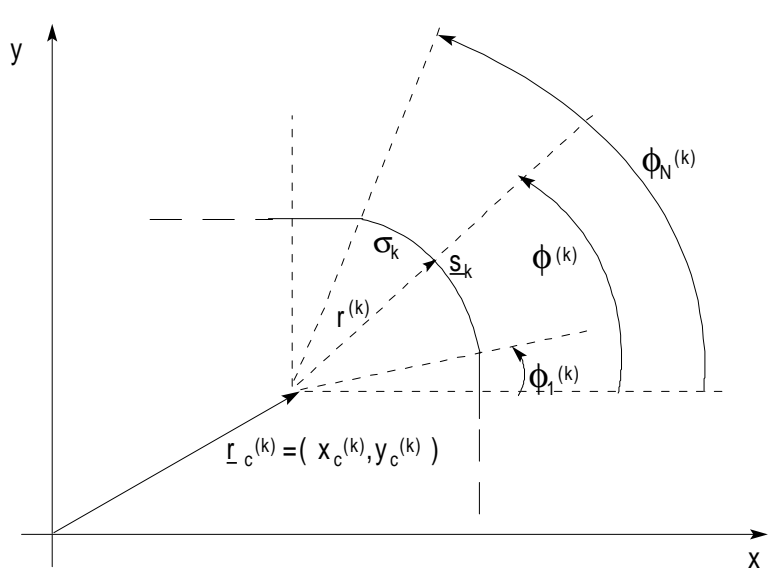

Fig. 1 - Local coordinate system relevant to eq. (12).

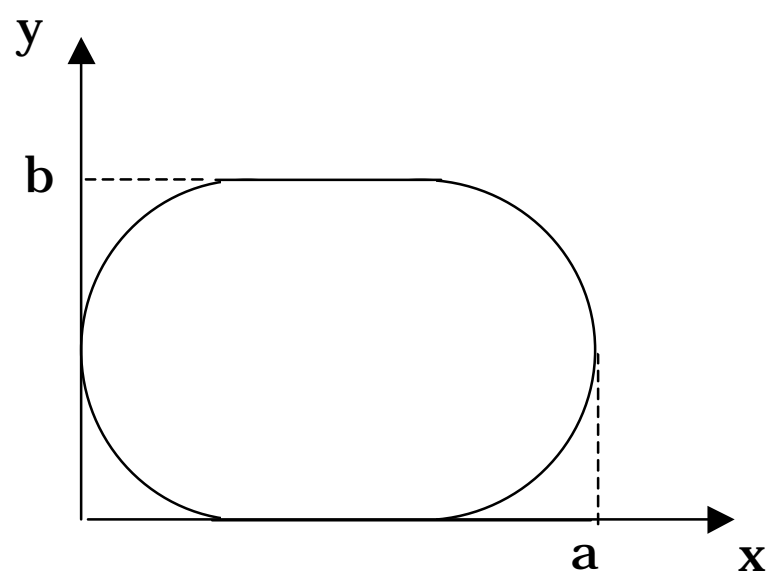

Fig. 3 - Stadium-shaped pipe $(a=1, b=0.7)$.

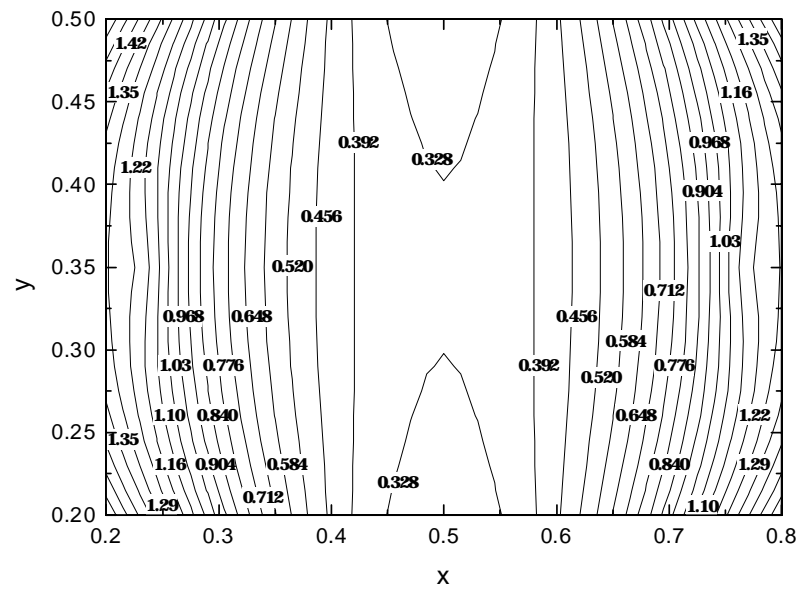

Fig. 5 - Stadium-shaped pipe. Coherent Laslett coefficient ( $1^{\text {st }}$ normal mode).

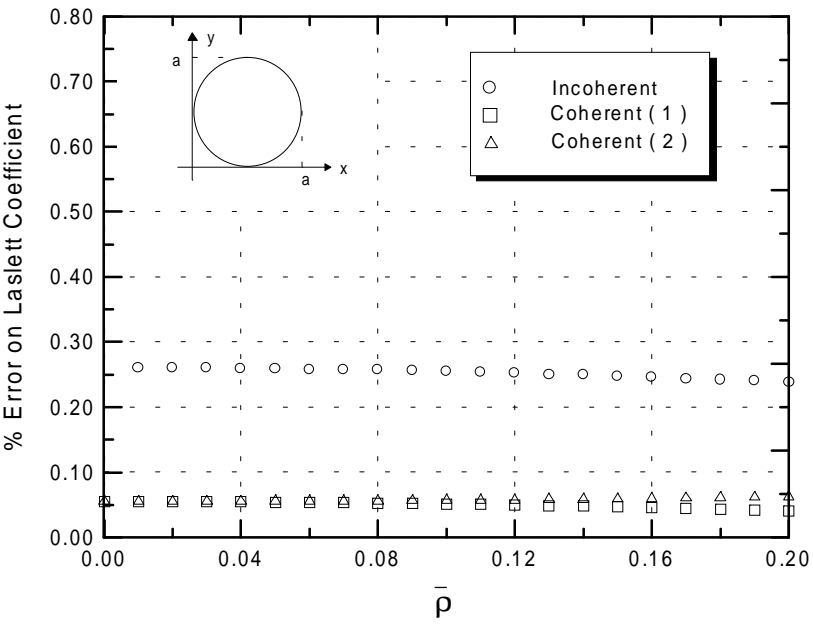

Fig. 2 - Circular pipe. Errors on Laslett coefficients vs. scaled radial distance, $\bar{\rho}=\frac{2}{a}\left[(x-a / 2)^{2}+(y-a / 2)^{2}\right]^{1 / 2}$.

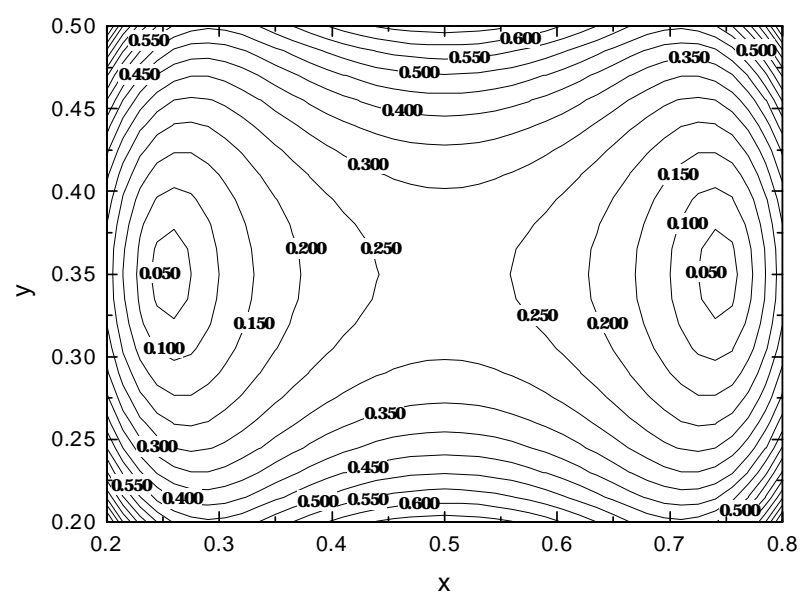

Fig. 4 - Stadium-shaped pipe. Incoherent Laslett coefficients (both normal modes).

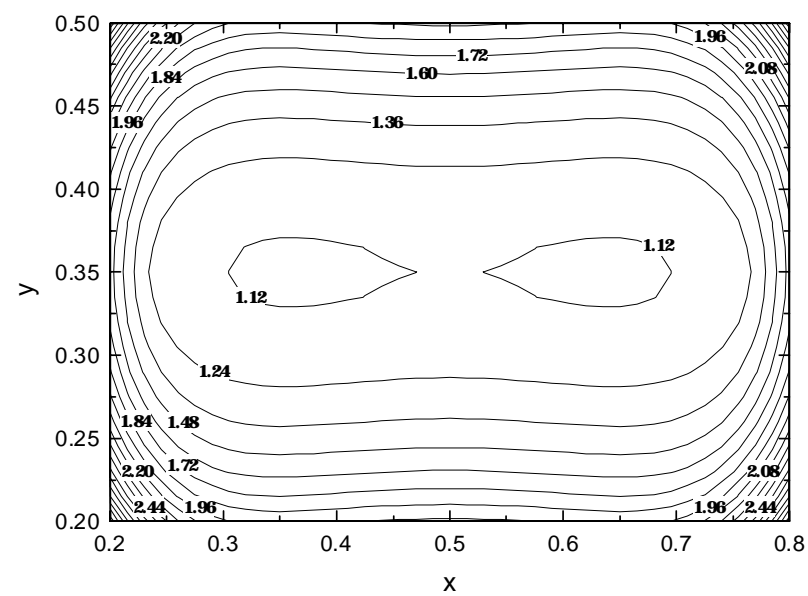

Fig. 6 - Stadium-shaped pipe. Coherent Laslett coefficient $\left(2^{\text {nd }}\right.$ normal mode). 